\title{
Labyrinthe
}

3| 1999

Numéro 3

\section{Images du corps et agressivité dans la classe}

Approche clinique des représentations d'enseignants et d'élèves d'école élémentaire

Sylvie Gérard

\section{OpenEdition}

1 Journals

Édition électronique

URL : http://journals.openedition.org/labyrinthe/60

DOI : $10.4000 /$ labyrinthe. 60

ISSN : 1950-6031

Éditeur

Hermann

Édition imprimée

Date de publication : 15 avril 1999

\section{Référence électronique}

Sylvie Gérard, «Images du corps et agressivité dans la classe », Labyrinthe [En ligne], 3 | 1999, mis en ligne le 04 mars 2005, consulté le 01 mai 2019. URL : http://journals.openedition.org/labyrinthe/60 ; DOI : 10.4000/labyrinthe.60

Ce document a été généré automatiquement le 1 mai 2019.

Propriété intellectuelle 


\title{
Images du corps et agressivité dans la classe
}

\author{
Approche clinique des représentations d'enseignants et d'élèves d'école \\ élémentaire
}

Sylvie Gérard

1 Les violences scolaires sont généralement considérées comme les manifestations exacerbées d'un mal de vivre social importé, de l'extérieur, au sein des établissements. Cependant, depuis peu, l'École n'apparaît plus comme totalement innocente. «Elle ne peut faire porter tout le poids de ses maux sur les seuls usagers ${ }^{2}$ ", nous dit-on. Afin d'éviter l'émergence d'agressions physiques et verbales en classe, tant de la part de l'enseignant que de l'élève, l'idée d'une relation pédagogique à interroger en permanence est de plus en plus fréquemment évoquée. Face au phénomène, le monde social et scientifique affirme ainsi la nécessité d'une réflexion qui ne s'arrêterait pas « aux portes de la classe mais inclurait au contraire l'acte pédagogique, dans sa quotidienneté relationnelle $^{3} »$. S'inscrivant dans cette perspective, une thèse tente de mieux comprendre les actes agressifs se produisant en classe à l'école élémentaire, par une approche clinique des représentations de l'enseignant et de l'élève.

2 Dans ce travail, l'agressivité est conçue comme une composante fondamentale de l'être humain. Sa présence dans la classe, lieu de rencontre de l'enseignant et de l'élève, y est donc considérée non seulement comme inévitable mais souhaitable, souhaitable lorsqu'elle est bien sûr orientée vers l'apprentissage. Par ailleurs, attentive aux nombreuses corrélations entre les violences scolaires et l'environnement, physique et humain, cette recherche soutient que les composantes matérielles et relationnelles d'un milieu peuvent se révéler pathogènes en n'autorisant pas l'individu à « aimer et haïr en même temps et accepter cette contradiction ${ }^{4} »$. Compte tenu, enfin, de la dimension subjective des agressions, cette thèse affirme que, pour saisir le phénomène de la violence à l'école, plutôt que d'étudier les faits en tant que tels, il est préférable de mettre en évidence les processus psychiques conduisant à leur désignation et à leur émergence. 
3 Cette étude avance ainsi l'hypothèse selon laquelle la façon dont l'enseignant et l'élève se représentent les conditions matérielles et relationnelles organisant la classe, contribue à réguler ou à accentuer les agressions scolaires. Elle affirme que le contenu figuratif de leurs représentations est structuré autour de significations tant sociales qu'individuelles. Elle soutient en outre que leur agressivité y est constamment présente. Elle prétend par ailleurs qu'un travail d'élaboration mentale vise à articuler, avec plus ou moins de bonheur, les registres affectif et social de ce contenu figuratif, et que ce dernier en porte l'empreinte. Elle assure enfin que l'agressivité des sujets trouve à s'exprimer dans l'agression lorsque cette articulation ne peut se faire. La vérification des hypothèses s'appuie sur une enquête réalisée auprès de 19 classes élémentaires ${ }^{5}$ de Seine-Saint-Denis. Le matériel recueilli entretient des liens étroits avec les hypothèses de départ. Il vise à faciliter l'accès aux représentations des sujets ${ }^{6}$. Il se compose de 19 plans et emplois du temps des classes, 21 entretiens $^{7}$ d'enseignants et 454 couples de dessins ${ }^{8}$ d'élèves ${ }^{9}$.

4 Cette recherche se soucie de déterminer la manière dont l'enseignant et l'élève passent d'une agressivité structurante et structurée, facilitant les apprentissages, à une agressivité destructrice et déstructurante, gênant, voire empêchant ceux-ci. Sa méthode d'analyse procède, tout d'abord, à une mise à plat du contenu figuratif des représentations ${ }^{10}$, suivie d'un repérage de leurs composantes matérielles et relationnelles, et de celles traversées par de l'agressivité. Vient ensuite la mise en perspective des contradictions, des effets de rupture ou de rapprochement, créés entre elles et avec les données issues des plans et emplois du temps. Pour finir, l'analyse des représentations des enseignants confrontée à celle des représentations d'élèves permet de voir les points de convergence ou de divergence éventuels.

5 L'exposé suivant s'intéresse plus particulièrement aux images corporelles qui hantent l'idée que se fait l'enseignant, ou l'élève, de la classe et des agressions qui s'y passent. Il procède pour cela en trois étapes. Tout d'abord, il précise le cadre général de la recherche. Puis, il décrit à la fois la présence constante de l'agressivité sur la scène pédagogique et la place du corps dans les représentations des sujets. Enfin, il évoque certains liens entre le fonctionnement de la classe et les agressions scolaires.

6 Aucun doute, la violence envahit l'École ! Certains faits, par leur aspect spectaculaire et leur corrélation avec des conditions sociales déplorables ${ }^{11}$, frappent évidemment l'imagination. Cependant, les attaques contre les biens et les personnes en milieu scolaire sont considérées comme résiduelles du point de vue policier et juridique. Par ailleurs, les situations évoquées ne s'avèrent pas toujours avoir des effets destructeurs dans la réalité. Certes, les propos traduisent un état de malaise. Les enseignants parlent d'incivilités, d'absence de travail, de motivation réduite. Leur souffrance, leur inquiétude et leur mécontentement se manifestent par une gêne, voire une impossibilité, à faire cours ainsi que par une remise en cause de leur capacité à enseigner. Les élèves évoquent l'irrespect, le manque d'écoute, le vol d'affaires... À leurs yeux, l'apprentissage en lui-même semble perdre toute signification. Les plaintes des uns et des autres portent sur ce qui vient perturber les individus ou empêcher le bon fonctionnement de l'institution et de l'apprentissage, et l'ambiguïté est très souvent de mise. Ainsi, " pour les adultes, l'agression réside d'abord dans la violence verbale. Mais aucun enfant ne cite cette dimension ; pas un ne considère qu'un "Nique ta mère! " ou un "Enfoiré! " soit une agression $^{12}$ ! «Ce qui est violence pour l'un ne l'est pas nécessairement pour l'autre. Une réflexion peut tout aussi bien être considérée comme un trait d'humour ou une attaque caractérisée. 
7 À l'évidence, ces situations qui vont des impolitesses au meurtre, semblent surtout « rassembler tout ce qui a trait à la lutte, au conflit, au combat, en bref à la part d'ombre qui toujours taraude le corps individuel ou le corps social ${ }^{13}$ ». Ainsi, derrière les propos se profilent toujours l'idée d'une intentionnalité des conduites, intentionnalité réelle ou imaginaire, ainsi qu'une résonance affective des faits, tant chez l'agresseur, la victime que le témoin. De ce fait, un décalage peut exister entre les intentions, connues ou inconnues, de celui qui agit et la signification donnée par la cible ou l'observateur. L'intention, plus ou moins consciente, voire inconsciente, avouée ou prêtée à autrui, est alors parfois plus agressive que le geste lui-même ou les effets de l'action. Par ailleurs, au-delà des faits, se retrouvent, dans les discours, des normes, des valeurs, des croyances mais aussi des peurs, des fantasmes, des angoisses et toute une gamme de sentiments divers.

8 Les discours sur les situations de violence scolaire expriment toujours le rapport personnel que les sujets entretiennent avec elles et avec la réalité qui les entoure ${ }^{14}$. Ils ont autant à voir avec des éléments du monde scolaire qu'avec ceux du monde intérieur des individus. Les propos tenus par l'enseignant et l'élève découlent de la représentation qu'ils se font de l'école, et plus particulièrement de l'apprentissage. Ils sont une reconstruction intellectuelle émanant à la fois de la singularité des sujets et de leur inscription sur la scène pédagogique. Afin de mieux comprendre la genèse de la " violence à l'école ", dans les représentations de la classe des adultes et des enfants, il parait cependant nécessaire de distinguer leurs aspects socioculturels de leurs aspects psychologiques et d'en estimer leurs poids respectifs. Une approche clinique, en postulant la présence d'un inconscient constamment à l'œuvre dans les conduites humaines, fait de l'enseignant et l'élève des sujets s'inscrivant dans une réalité de classe, produit de combinaisons incessantes entre des composantes imaginaires et réelles propres à chacun. Ce faisant, elle place le phénomène de la « violence à l'école » dans le registre des expériences culturelles individuelles : la culture étant entendue ici comme la tradition dont chacun hérite, sorte de fond commun allant s'élargissant du noyau familial à l'humanité, et sur lequel tout sujet s'appuie afin d'être lui-même.

9 Une telle approche conduit en outre à se pencher sur le problème de l'agressivité humaine. En effet, les exactions scolaires qui concernent aussi bien l'élève que l'enseignant, ne sont pas toutes sauvages et destructrices dans les faits. Mais elles perturbent toujours les sujets. Elles portent toujours atteinte à leur intégrité, atteinte directe ou indirecte, physique ou psychique, effective ou potentielle. Certes, ces conduites sont parfois des violences, c'est-à-dire des « actes destructeurs appliqués aux corps ou aux biens matériels ». Mais elles constituent plus largement des agressions, c'est-à-dire des « actes porteurs d'agressivité humaine mais pas nécessairement destructeurs pour les corps et les biens matériels ». Vouloir étudier la « violence à l'école » revient à considérer la place de l'agressivité humaine dans la relation pédagogique.

10 L'agressivité est cette " tendance ou ensemble de tendances qui s'actualisent dans des conduites réelles ou fantasmatiques, celles-ci visant à nuire à autrui, le détruire, le contraindre, l'humilier ${ }^{15} . .$. » Elle reconnaît ainsi l'autre en tant que sujet. Les agressions scolaires représentent, dans leur très grande majorité, des comportements sociaux mettant en relation des individus au sein de l'institution. D'un point de vue psychologique, elles relèvent donc avant tout du champ d'observation de l'agressivité humaine, non de celui de la "violence fondamentale », pour reprendre l'expression de Bergere ${ }^{16}$. La " violence fondamentale » qui touche aux fondations de toute structure de la personnalité, est une composante instinctuelle innée qui ne comporte en effet aucune 
intention de nuire même si son résultat est destructeur. Lorsque la "violence fondamentale " d'un sujet se manifeste, seul compte, pour lui, son intérêt immédiat. L'autre n'a pas d'importance. Il ne s'agit pas de lui nuire. Il s'agit simplement de se protéger. Nécessaire à la survie, la "violence fondamentale " relève du groupe des instincts de vie primitifs. Sa trace se retrouve, en conséquence, très difficilement à l'état pur dans les comportements, à l'exception du passage à l'acte ${ }^{17}$ ou du règlement d'un problème de l'ordre de la survie ${ }^{18}$. Très tôt, en effet, l'être humain prend conscience des conséquences qu'entraînent de telles réactions sur son entourage. Ce faisant, il retire de ces actes un certain degré de satisfaction et de culpabilité. Il « apprend » à jouer de sa violence. Il « apprend » l'agressivité.

11 L'agressivité repose, en fait, sur une activité mentale élaborée. Elle trouve d'autres modalités d'expression que la destruction. Elle peut être orientée vers des buts socialement acceptables, notamment vers l'apprentissage ${ }^{19}$. Sa capacité à se déplacer, à se symboliser, à se sublimer, ou à atténuer ses effets, en font une composante fondamentale de l'être humain. Freud ne disait-il pas que « nos meilleures vertus sont nées comme formations réactionnelles et sublimations sur l'humus de nos plus mauvaises dispositions ${ }^{20}$ » ? Il précisait aussi toutefois, à propos de l'agressivité, qu'« il n'est pas facile de concevoir comment on peut s'y prendre pour lui refuser satisfaction [...] si on ne compense pas ce refus de manière économique ${ }^{21} »$. Comprendre le sens de l'émergence des comportements sauvages et destructeurs à l'école élémentaire passe sans doute, pour partie, par une meilleure définition de sa place dans le fonctionnement des classes, ou plus précisément de la place qu'elle occupe dans les représentations de l'enseignant et de l'élève.

12 Issue du corps, en tant que pulsion, l'agressivité traverse en permanence ce dernier. Elle utilise sa musculature, ses orifices pour se projeter vers l'extérieur. Elle se dirige alors, de manière plus ou moins perceptible, plus ou moins détournée, vers d'autres corps qu'elle atteint à leur surface, sur la peau. L'agressivité pénètre les corps, physiquement ou symboliquement, y imprimant son empreinte. Empreinte externe, celle du coup porté. Empreinte interne lorsque l'agression touche au plus profond de l'individu ou bien lorsque dans l'impossibilité de s'extérioriser, l'agressivité va s'attaquer au corps même de celui qui la sécrète.

13 À l'école, l'agressivité ne s'attache pas seulement aux corps dans leur aspect charnel mais aussi, et surtout, aux corps investis par l'enseignant ou l'élève. Elle touche à ces corps de l'imaginaire que chacun porte en soi, celui qu'on imagine avoir, celui qu'on croit voir chez l'autre, ces corps porteurs d'une histoire, de fantasmes, de peurs, de désirs... Ces images corporelles, si différentes et si proches à la fois des corps matériels, synthétisent les expériences émotionnelles de l'adulte et de l'enfant. Elles expriment leur relation à eux-mêmes et aux autres, et donc au monde scolaire qui les entoure. Elles bouleversent parfois aussi leurs certitudes, ravivent leurs fantasmes agressifs, s'opposent à leurs désirs...

14 Les dessins et les discours présentent de nombreux éléments agressifs, réels, supposés, redoutés, minimisés ou niés, voire totalement fictifs, se situant dans le registre du manifeste ou du symbolique. Leur présence constitue autant de traces confirmant l'existence de l'agressivité humaine au sein des représentations de la classe de l'enseignant de l'élève. Plus ou moins repérables, cohabitent en permanence des traces de nuisance, de contrainte et de destruction. Et toutes ramènent inévitablement à des références corporelles. 
15 En classe, certaines situations s'avèrent perturbantes sans que pour autant les individus soient touchés physiquement ou les éléments matériels détruits. La situation de classe apparaît préjudiciable à l'élève, à l'enseignant. Ils s'en plaignent : trop ou pas assez de travail, de bruit... Parallèlement, chacun contribue à son mauvais fonctionnement : refus de toutes sortes, joutes verbales... Exprimant parfois ouvertement leur intention de nuire, adulte et enfant font alors obstacle au déroulement normalement attendu de la classe. Ils interdisent l'instauration de relations interpersonnelles de qualité. Au détour des entretiens, des dessins, surgissent des scènes d'opposition aussi bien dans la relation maître/élève qu'entre élèves. Dans une classe « qui se passe bien », un affrontement entre élèves n'est jamais totalement exclu (fig. 1). Les descriptions, adultes et enfantines, font continuellement état de comportements perturbateurs ${ }^{22}$, quand elles ne font pas référence à des conduites pour le moins inadaptées ou incongrues ${ }^{23}$. Comportements malfaisants, malveillants ou impressions d'évoluer dans un environnement néfaste, nocif sont l'envers et l'endroit d'une même médaille, celle de la nuisance.

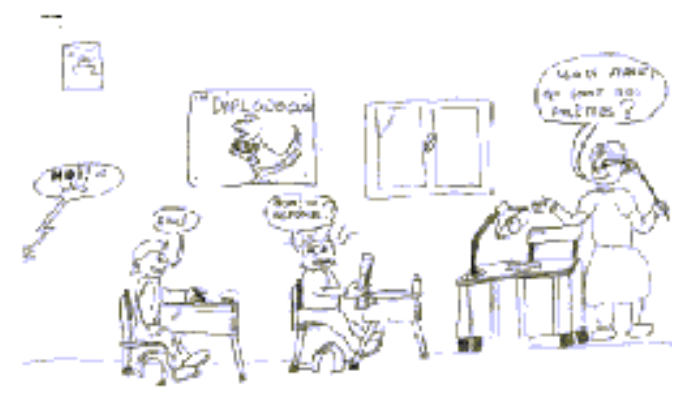

Fig. 1 : l'agressivité humaine : une présence constante dans la classe (dessin de CM 2 d'une classe qui « se passe bien »)

16 En infiltrant les aspects matériels ou institutionnels ${ }^{24}$ de la représentation de la classe, les images corporelles participent à ces perturbations. Les corps se refusent, s'opposent, se ferment, revendiquent, se plaignent... La bouche, avec sa parole abondante, vole la place de l'autre. Alors, on l'empêche de s'exprimer. On refuse de l'écouter. Pour l'enseignant, l'élève est bavard. Mais la réciproque est vraie aussi. Dans la classe qui va mal, le maître parle beaucoup. Il abrutit d'ordres, de savoirs, de questions... Tandis que sa parole envahit l'espace, sa bouche s'ouvre démesurément découvrant parfois quelques dents inquiétantes. Parler n'est cependant mal vécu que lorsqu'un risque potentiel de dérive vers le bruit, la cacophonie et la non-maîtrise de la classe l'accompagne dans l'imaginaire.

17 Le bruit est la nuisance première à l'école, mais elle n'est pas la seule. D'autres perceptions sensorielles désagréables renforcent les sentiments de nuisance. Le nez, siège de l'odorat, est ainsi souvent présent dans les métaphores corporelles : on ne peut pas se sentir mais en même temps on sent bien que... Mais au-delà des figures de style, il est aussi de véritables odeurs dans la relation éducative qui viennent renforcer les sentiments agressifs ressentis face à certains enfants : " Elle euh elle SENTAIT AFFREUSEMENT MAUVAIS, et la p'tite, elle est habillée comme si elle sortait de la poubelle. Et elle est jamais lavée. Donc, euh bon c'est une gamine quand même euh... Tout va ensemble quoi! Quelle attitude tu dois avoir ${ }^{25}$ ?" Les odeurs corporelles compromettent l'environnement scolaire tout autant que les bavardages. En rendant la vie de classe pénible, les corps contribuent à dessiner un espace pédagogique potentiellement nuisible. Et les perturbations qu'ils 
occasionnent, bénignes dans les faits, n'en sont pas moins ressenties fort agressivement parfois.

Outre la nuisance, la destruction se manifeste aussi sur la scène pédagogique. Physique, elle atteint l'intégrité des corps et des biens. La parole et le trait évoquent des meurtres, des suicides, des bagarres et plus généralement des contacts corporels directs ou indirects ${ }^{26}$, ainsi que des objets ${ }^{27}$ abîmés, salis ou anéantis. Psychique, la destruction touche à l'intégrité des esprits. Certains actes, propos, ou situations contribuent à faire apparaitre autrui, ou soi-même, comme inférieur, méprisable, sans valeur. D'autres suscitent des sentiments d'affront ou de honte. Ces destructions, réelles ou potentielles, constamment présentes dans les représentations de la classe, s'accompagnent d'images corporelles.

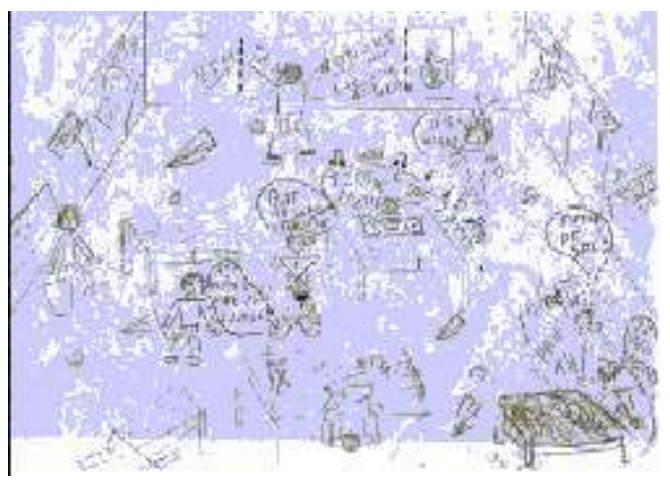

Fig. 2 : l'agressivité humaine : une présence manifeste dans la classe qui « se passe mal » (dessin de CM2)

19 Les corps provoquent, ou supportent, l'agression, tout autant qu'ils expriment leurs souffrances intérieures. La bouche introduit l'humiliation, la destruction psychique, dans l'espace pédagogique. Les moqueries, les remarques acerbes, les insultes et les propos racistes peuvent être fortement marqués (Fig. 2), au travers de tags ou d'affirmations. «Là, j'vois Farid qui habituellement euh... pose pas d'problèmes eh bien! tout à l'heure on est v'nu m'dire "Oui ! Farid il a dit qu'il aimait pas les noirs" euh ...8 ». Mais, ils peuvent aussi être plus insidieux : «C'est mon moumou de service. Il est adorable ! C'est un enfant de couleur. Mais bon euh... il est adorable ${ }^{29}$ ! $»$ Sur sa peau, le corps porte aussi parfois les stigmates de l'agression morale, psychique, qui lui a été faite... Et l'enseignante peut y lire la preuve culpabilisante de sa propre agressivité : «Écoute j'me suis pas trompée, j'me suis mise à crier et à mesure que j'criais j'VOYAIS les plaques qui sortaient, qui sortaient...»

20 Les corps entrent en contact directement, et indirectement lorsqu'un objet devient le trait d'union d'un corps à corps plus distancié. Les élèves, surtout, se touchent par les coups. Ils se rentrent dedans. Leur puissance destructrice, très affirmée graphiquement, se retrouve aussi dans les entretiens : «C'était pas une p'tite bagarre, un p'tit coup d'pied, c'était... ils s'claquaient la tête contre le sol et tout ${ }^{30}$ ! ». Souvent, des commentaires relativisent ces actes, nient leur caractère, voire l'ignorent ou le valorisent : « ...quand il frappe, il fait un peu mal. Et il sait se battre. J'l'ai vu faire dans la cour. Euh...il est pas manchot ${ }^{31}$. » Si les coups portés ne font pas mal ou ne cherchent pas à faire mal, ces corps à corps sont ludiques. L'idée d'enfants agressifs est alors repoussée. Pourtant, à travers ces «jeux", la destruction peut à tout moment resurgir, et conduire vers ces corps enchevêtrés des bagarres, agglutinés en tas infâmes, en mêlées confuses (Fig. 2). Est-ce par hasard si les élèves agressifs donnent l'impression à l'enseignant, de "faire clan ", 
expression qui évoque symboliquement cette réunion des corps? N'est-ce pas la peur du contact corporel, et de ces dérives potentielles, qui l'amène à isoler ces enfants en classe ? Lorsque le maitre sépare des corps qui auraient tendance à se rejoindre, il évite que certaines menaces, trop inquiétantes, ne conduisent aux destructions physiques qu'elles laissaient supposer. «Mais Bilé aussi est assez, assez agressive euh, euh ça commence par euh l'agression euh, euh ver, verbale...et des fois ça passe euh des cas euh dans le ... ${ }^{32}$ ». Cependant, parallèlement il refuse aux élèves une place dans la communauté scolaire et s'en fait le reproche. Atteint moralement, il se sent honteux de n'être pas à la hauteur et cherche à rapprocher, ne serait-ce que momentanément, ceux qu'il a écartés. S'instaure ainsi, sur fond de destruction à la fois physique et psychique, un jeu étrange de séparation/réunion des corps au sein de la classe.

Si la nuisance et la destruction infiltrent en permanence les représentations de l'espace pédagogique, la contrainte aussi. Les évolutions corporelles s'y trouvent limitées. Des pressions morales, souvent traduites par un sentiment d'obligation, transparaissent. Dans les dessins, les personnages, parfois ligotés, voient très souvent leurs mouvements limités par un tiers. Celui-ci les oblige à se déplacer vers un point précis, ou à attendre un ordre sans bouger. Les discours, de leur côté, laissent apparaître un ensemble de devoirs, plus ou moins bien acceptés, auxquels nul n'est censé échapper. Des situations de contention physique, de surveillance et de contrôle sont évoquées. Un enseignant de CE1 précise ainsi que « Oh ! ben, bien souvent, j' suis obligée d'intervenir en, en, en rattrapant le gamin agressif, puis en, en l'tenant et en disant «Eh ! Oh ! tu t'arrêtes ! Euh... « Tu te calmes! » Enfin, bon... faut le faire reculer parc'que... obligée d'l'empoigner, en fait euh... ».

La classe est un lieu de contrainte pour les corps et les esprits. Et les images corporelles participent, une fois de plus, à cette situation. Mais la contrainte n'est pas toujours mal vécue. La bouche véhicule, nous l'avons vu, un ensemble de sous-images intimement liées à l'agressivité : la peur, la haine, la souffrance, la dévoration, l'ensevelissement sous un flot de parole, la perte de sa place, le refus du contact, la destruction de l'image de soi... Sans compter le non-dit ou l'interdit du dire... Elle est gestionnaire de l'agressivité, celle que l'on sent monter en soi, celle réellement exprimée ou fantasmatiquement vécue. On recourt aux mots pour calmer les esprits. On instaure des tours de parole ou le silence. On laisse s'exprimer. On utilise la voix à d'autres buts. On chante... On tente de contrôler et de se contrôler afin de ne plus agresser.

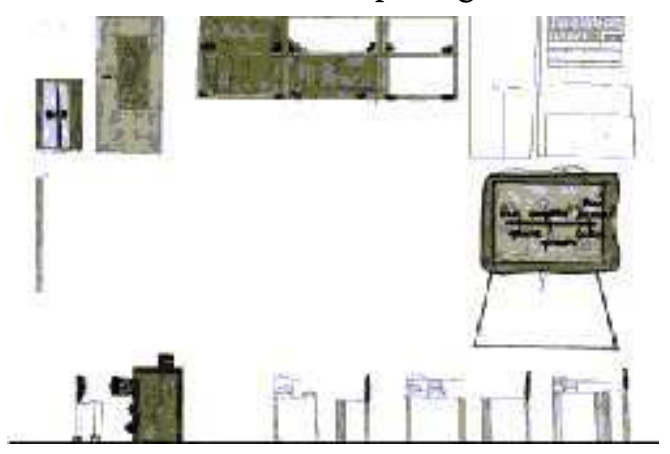

Fig. 3 : la classe qui « se passe bien » ou la question de l'absence des corps (dessin de CE1)

La contrainte est désirée par les enfants. Leurs représentations de la classe qui va bien sont très traditionnelles. Chacun est à sa place : le maître au bureau ou au tableau, les élèves à leur table. Les corps sont pétrifiés, ou bien absents. Les représentations vides de 
personnages (fig. 3) caractérisent en effet ces dessins. Les corps dynamiques n'illustrent que les fonctionnements pédagogiques défectueux. Et, l'interdit officiel du toucher est loin d'y être respecté. Dans la relation pédagogique dégradée, si les corps sont plus souvent présents, les contacts corporels aussi. Le désir enfantin de contraintes trouve un écho dans celui de leurs maîtres pour qui le meilleur moyen d'éviter la destruction consiste à figer les corps. Certes le mouvement entrâne nécessairement une possible dérive vers la brutalité. Cependant, comment vivre sans bouger ? Bouger sans toucher l'autre est une obligation, aussi, la solution est-elle d'autoriser peu de déplacements en classe, ou de les codifier. On se déplace en petits groupes. On ne s'agglutine pas. Si l'on se retrouve un instant à la bibliothèque, c'est pour vite retourner à sa place et à son isolement. Le rapport du maître, et de l'élève, à ces contraintes reste toutefois toujours ambigu. Désirées parce que nécessaires pour prévenir des exactions toujours possibles, elles leur deviennent en effet très vite insupportables ${ }^{33}$.

Les discours des enseignants, les dessins d'élèves présentent la confrontation à l'agressivité humaine sur la scène pédagogique comme inévitable. Ces images agressives de destruction, de contrainte et de nuisance qu'ils développent, tiennent cependant tout autant à leur monde intérieur, à leurs fantasmes, qu'à la réalité. Graphiquement, les cannibales, les lions, les tigres, les araignées, les gibets, les guillotines, les armes en témoignent. Verbalement, les métaphores ${ }^{34}$ et les traits d'humour relèvent d'une même logique. Ces images symboliques renvoient aux «matrices productives d'intentions agressives " de Lacan. Des idées de castration, d'éviration, de mutilation, de démembrement, de dislocation, de dévoration, d'éventrement et d'éclatement des corps traversent la représentation de la classe. Elles sont le filtre par lequel l'enseignant, et l'élève, jugent de l'agressivité de la situation pédagogique. Elles impulsent leurs agressions. Ainsi en est-il des classes «agressives» qui «bouffent» le maître. Ni totalement éden, ni totalement géhenne, leurs représentations de la classe évoluent cependant surtout dans une tierce zone où l'ombre borde sans cesse la lumière. Dans le clair-obscur de leur espace pédagogique, se tapit une agressivité toujours prompte à investir le devant de la scène, mais aussi apte à assurer la vie scolaire et les apprentissages.

À leur manière, les enfants esquissent une classe où l'agressivité, loin d'être interdite d'expression, peut trouver à s'investir dans l'apprentissage, une classe avec «des dispositifs, des positions pédagogiques, qui rendent parfois possible que ça grandisse, que ça apprenne ${ }^{35}$ ». Certes, la consigne négative favorise l'expression des sentiments négatifs de l'élève, et conduit celui-ci à des mises en scène agressives plus nombreuses. Cependant, avec la consigne positive, l'importance numérique de ces dernières est loin d'être nulle. En effet, en moyenne, près de deux indicateurs agressifs ${ }^{36}$ y sont relevés par dessin. Quelle que soit la consigne, l'agressivité constitue en fait une constante pédagogique, mais une constante dont la forme diffère selon la consigne, ou le jugement d'agressivité porté par l'enseignant sur l'auteur du dessin.

26 Tout d'abord, pour la classe qui se passe bien, les traces agressives symboliques deviennent quasi inexistantes. Par ailleurs, $15,9 \%$ des indicateurs font état de destruction, physique ou psychique, or ce taux atteint $74,55 \%^{37}$ dans les figurations de la classe qui « se passe mal «. Ce renversement de situation s'accompagne d'un mouvement inverse, quoique moins accentué, au niveau des indicateurs de nuisance et de contrainte. Lorsque les élèves dessinent une classe qui va mal, le pourcentage des premiers passe de $31,80 \%$ à $14,30 \%$, celui des seconds de $52,30 \%$ à $11,15 \%$. Enfin, la qualité de ces 
contraintes et nuisances change. Les marques d'opposition et d'obligation augmentent avec la consigne négative, celles d'inadaptation et de surveillance diminuent. Une évolution similaire, plus faible, s'observe sur les dessins des élèves jugés « agressifs » par leur enseignant. Elle s'explique par le fait que, indépendamment de la consigne de départ, leurs productions évoquent plus de destructions que celles de leur camarade. Bien que les regards enfantins sur la scène pédagogique varient sous les effets du monde psychique destructeur qui habite certains, la classe " qui se passe bien " est vécue moins agressivement. Elle ne détruit pratiquement plus, psychiquement ou physiquement, les sujets. Elle accorde une place à leurs comportements perturbateurs et incongrus, tout en les surveillant étroitement. L'agressivité humaine est présente mais canalisée.

27 Par ailleurs, en traçant ces situations et ces fantasmes agressifs, les enfants dessinent en contre-jour, des espaces, des temps, des modalités relationnelles avec les autres et le savoir. Ces circonstances pédagogiques évoluent, elles aussi, selon la consigne, et sur cette évolution s'enracinent les comportements agressifs. Quand ça va mal, la représentation du savoir devient ainsi, trop simpliste ou trop complexe. Le matériel pédagogique se dégrade, ou n'est plus accessible. La leçon s'éternise ou n'a pas lieu. L'évolution des dessins d'enfants montre qu'à ne pas occuper les esprits, ou à mal le faire, le risque est grand de voir les corps et la destruction envahir la classe.

Les descriptions de situations d'agression, les portraits d'enfants jugés perturbateurs, agressifs, voire violents, les évocations d'un sentiment de nuisance ou de contrainte tiennent une place peu importante dans les discours magistraux ${ }^{38}$, sans en être totalement absents. Ainsi, alors que 100 élèves sont plus particulièrement décrits, un jugement explicite d'agressivité n'est porté que sur 35 d'entre eux. Malgré tout, les différentes images agressives exprimées et leur éventuelle évolution vers une solution positive peuvent se comprendre au travers de certains décalages ou chevauchements catégoriels, si l'on peut dire. En effet, la structuration interne des entretiens laisse apparaitre différentes unités de sens se répartissant en cinq catégories : les constats, les analyses, les certitudes, les sensations et les désirs.

Un enseignant de CM2 affirme ainsi la nécessité qu'ont les hommes de se parler. Puis, il constate que dans la classe « en fin de compte, l'échange, il est toujours avec le maître «. Il évoque alors son désir d'apprendre aux élèves " à s'exprimer, apprendre à... parler, mais à parler sur des choses, des vraies...». Mais lorsqu'il rappelle une confrontation ancienne à une classe de $\mathrm{CP}$ dans laquelle il se sentait en difficulté, il constate : " Je ne pouvais plus expliquer les choses comme euh avec euh les grands. Y avait plus cette, je pouvais plus communiquer d'la même manière, expliquer les mêmes choses. " ou bien encore « Ben on, on essayait bien, quand même d'passer par la parole... Mais euh... ouais... oh, oui ! C'était, c'était difficile». Au fil de l'entretien, il finit en fait par analyser la situation, la rationaliser avec un « Parc'que j'avais des enfants très difficiles, très agressifs ! Alors là qui se tapaient d'sus hein! ». L'agressivité des enfants vient marquer, renforcer en quelque sorte, l'impossibilité d'établir ce dialogue si essentiel à ces yeux.

Dans les discours, la réalité de la classe se combine avec certaines scènes imaginaires. En trouvant un étai dans la classe, ces dernières poussent les enseignants à prendre comme agression ce qui n'est parfois qu'une remise en cause de leurs certitudes ou de leurs désirs. Certes, l'agressivité humaine se niche au cœur des propos. Elle ne surgit cependant que lorsque les normes, les valeurs ou les fantasmes des sujets, en bref, lorsque leur monde intérieur entre en conflit avec la réalité extérieure, ou bien que les conflits intrapsychiques deviennent trop forts. À cet instant, l'enseignant devient incapable de 
négocier les situations. Son agressivité, destructrice et déstructurante, occupe alors une place prépondérante sur la scène pédagogique. Elle se manifeste soit par des paroles ou des actes agressifs, soit par des jugements d'agressivité parfois surprenants : "J'en ai une qui est dans son monde, qui est assez fermée, qui est assez agressive, mais bon... C'est son tempérament quoi ! Elle est, j'veux dire, on lui dit quelque chose... c'est comme si elle entendait pas. Faut lui répéter trois ou quatre fois pour que elle le, elle le fasse. C'est une agressivité intérieure en fait! Elle euh elle se manifeste pas directement. Elle va pas aller agresser un enfant. Mais bon, quand même on la sent agressive à l'intérieur d'elle-même si vous voulez ${ }^{39}$...»

31 Sous le crayon, derrière les mots, le message est clair. L'école, ne fera jamais l'économie de l'émergence, sur la scène pédagogique, d'agressions tant magistrales qu'enfantines, même si « requise à la conciliation des rapports humains, il lui revient de réduire, autant que possible, ce qui peut fabriquer de toutes pièces de la destructivitéte ${ }^{40}$. Et les regards portés sur les corps, au même titre que ceux sur le temps, l'espace, les savoirs ou les relations, mais parfois plus encore, entretiennent des liens étroits avec l'émergence des agressions en classe. Les corps charnels véhiculent l'agressivité de l'enseignant et de l'élève. Ils participent aux agressions surgissant en classe et donc au phénomène des " violences scolaires «. En outre, nombre de représentations corporelles, le plus souvent imaginaires, fantasmées, reflètent les craintes et les désirs mais aussi les attentes, les normes et valeurs de chacun. En bref, constamment, des images corporelles traversent et travaillent du dedans la relation pédagogique.

32 Certains mouvements psychiques président plus particulièrement au surgissement des agressions scolaires ainsi qu'à l'ambiguïté de leur désignation. Corps imaginaires de géants ou de nains, corps qui se refusent, qui s'opposent, qui se ferment, qui revendiquent, qui se plaignent... Ces images corporelles, en décalage, voire en opposition avec les corps attendus, envahissent l'espace d'apprentissage qu'est la classe et contribuent bel et bien à des impressions d'agression, à des sentiments agressifs, qui finissent parfois par prendre forme au travers des corps réels. Aucun doute, un ensemble de significations se dégage des potentialités destructrices des corps, potentialités réelles et imaginaires, et donne au phénomène de la violence à l'école une portée symbolique, une portée « culturelle « au sens freudien du terme. La relation pédagogique ne se réalise pas seulement au niveau de l'objectivement perçu, elle relève aussi du registre de la subjectivité. Elle n'est pas simplement intellectuelle, elle est aussi charnelle. À ne pas tenir compte de cela, l'École ne peut que voir le corps de l'élève, ainsi que celui de l'enseignant, au mieux, déranger l'apprentissage, au pire, se réintroduire douloureusement dans le champ éducatif au travers d'agressions manifestes.

\section{BIBLIOGRAPHIE}

ANZIEU Didier, Le Moi-peau, Paris, Dunod, 1995 ( $2^{\mathrm{e}}$ éd.).

BALIER Claude, Psychanalyse des comportements violents, Vendôme, PUF, 1995.

CHILAND Colette, YOUNG J. Gérald (dir.), Les Enfants et la violence, Vendôme, PUF, 1997. 
DEBARBIEUX Éric, La Violence dans la classe, Condé-sur-Noireau, ESF, 1990.

DOLTO Françoise, L'Image inconsciente du corps, La Flèche, Le Seuil, 1992.

FREUD Sigmund, « Au delà du principe de plaisir », Essais de psychanalyse, Paris, Payot, 1981.

LACAN Jacques, « L'agressivité en psychanalyse », Écrits, Paris, Le Seuil, 1966.

LAGACHE Daniel, Agressivité structure de la personnalité et autres travaux, Paris, PUF, 1982.

Les Cahiers de la Sécurité Intérieure, $n^{\circ} 15,1^{\mathrm{er}}$ trimestre 1994.

POSTIC Marcel, La relation éducative, Paris, PUF, 1992 (5éd.).

PUJADE-RENAUD Claude, Le Corps de l'élève dans la classe, Aubenas, ESF, 1983.

PUJADE-RENAUD Claude, Le Corps de l'enseignant dans la classe, Aubenas, ESF, 1984.

Revue Française de Pédagogie, nº 123, avril-mai-juin 1998.

\section{NOTES}

1. Ces résultats sont extraits d'une thèse en Sciences de l'Éducation, actuellement en cours et intitulée La place de l'agressivité dans le fonctionnement de la classe. Approche clinique des représentations d'enseignants et d'élèves de CE1 et de CM2.

2. Mireille Cifali, Le Lien éducatif : contre-jour psychanalytique, Vendôme, PUF, 1994, p. 107.

3. Bernard Charlot, Jean-Claude Émin (coord.), Violences à l'école. États des savoirs, Paris, A. Colin/Masson, 1997, p. 13.

4. Donald W. Winnicott, Déprivation et délinquance, Saint-Amand-Montrond, Payot \& Rivages, 1994, p. 117.

5. Soit 11 CE1, 8 CM2.

6. Le recours aux plans et emplois du temps des classes facilite en effet l'étude du rôle du temps et de l'espace dans le fonctionnement de la classe. Le langage et le graphisme constituent par ailleurs deux moyens de communiquer à autrui ses représentations.

7. Soit 11 en CE1 et 10 en CM2 (Certaines classes ayant deux maîtres, le nombre d'enseignants est supérieur à celui des classes).

8. Soit 259 couples en CE1 et 195 en CM2.

9. Les entretiens individuels, semi-directifs, débutent par la question « Comment ça se passe pour vous dans une classe? ». Les dessins, réalisés en passation collective, à une semaine d'intervalle, répondent à « Dessine une classe où ça se passe bien » et à « Dessine une classe où ça se passe mal ».

10. Par une analyse de contenu des entretiens et des dessins.

11. Chômage, retard scolaire, délinquance...

12. Le Monde de l'Éducation $\mathrm{n}^{\circ} 226$, mai 1995 , p. 43.

13. Michel Maffesoli, « Violence », Les Notions philosophiques, Vendôme, PUF, tome II, 1990.

14. Les comportements repérés étant toujours considérés par les sujets comme inappropriés à leurimage de l'école.

15. Jean Laplanche, J.-B. Pontalis, Le Vocabulaire de la psychanalyse, Vendôme, PUF, $9^{e}$ édition, 1988, p. 13.

16. Jean Bergeret, La Violence fondamentale, Ligugé, Dunod, 1984.

17. Dans l'agression relevant du passage à l'acte, la pensée et le langage sont remplacés par l'acte. 
18. Le fameux « C'est lui ou moi » où l'objectif est de se protéger, ce qui peut advenir à l'autre n'entrant pas en ligne de compte.

19. Ne dit-on pas attaquer un problème, se battre contre une traduction?

20. Sigmund Freud, "L'intérêt de la psychanalyse ", Résultats, idées, problèmes, Vendôme, PUF, tome I, 1995, p. 213.

21. Sigmund Freud, Malaise dans la civilisation, Vendôme, PUF, 1971.

22. Tels que chanter ou siffloter pendant un cours.

23. Comme monter sur la table, dormir ou téléphoner.

24. Par exemple, des odeurs nauséabondes, les dimensions du local, le manque de matériel ou bien la lourdeur des programmes.

25. Enseignant CE1.

26. Tels que tirer les cheveux ou les oreilles, lancer un objet sur autrui, etc.

27. La classe pouvant être un objet au même titre qu'un cahier, un meuble ou un vase.

28. Enseignant de CE1.

29. Enseignant de CM2.

30. Enseignant de CM2.

31. Enseignant de CM2.

32. Enseignant de CE1.

33. «Et les autres tables ce sontdes tables pour enfants! avec le siège qui est fixé à la table. C'est à dire que quand il est là d'dans, crac ! il bouge plus ! Bon, y a pas les chaînes, ils les ont enl'vées ». Enseignat de CM 2.

34. "J'ai l'habitude de dire aux jeunes qui arrivent et qui se font plein d'illusions, soit qu'ils sortent de l'École Normale, soit qu'ils ont pas eu de formation ; je leur dis : euh... la première chose à savoir quand on entre dans une classe c'est qu'on entre un peu dans la cage aux fauves... » Enseignant de CE1.

35. Mireille Cifali, op. cit., Vendôme, PUF, 1 ère édition, 1994, p. 73.

36. Dans le champ de la destruction se trouvent les indices d'atteinte aux biens ou aux personnes, les menaces, humiliations et inquiétudes ; dans celui de la nuisance les marques d'opposition, d'inadaptation, de perturbation, d'incongruité ou les plaintes ; dans celui de la contrainte celles de contention, d'obligation ou de surveillance. La moyenne est 1,81 : soit 824 indicateurs pour 454 dessins. Elle n'est évidemment pas comparable à celle de la consigne négative, qui est de 18,48 : soit 8392 indicateurs pour 454 dessins.

37. Quantification et fréquence des indicateurs agressifs dans les dessins, respectivement pour « la classe où ça se passe bien » / « la classe où ça se passe mal »

- nuisance : 262 cas $(31,8 \%) / 1200$ cas $(14,3 \%)$

- contrainte : 431 cas $(52,3 \%) / 936$ cas $(11,15 \%)$

- destruction physique : 35 cas $(4,25 \%) / 4512$ cas $(53,77 \%)$

- destruction psychique :96 cas (11,65\%) / 1744 cas $(20,78 \%)$

- total : 824 cas (100\%) / 8392 cas (100\%)

38. Ainsi, deux enseignants ne font aucune référence à des situations d'agression, pour autant, l'agressivité trouve toujours à transparaître dans leurs discours au travers des sentiments qu'elles expriment.

39. Enseignant de CE1.

40. Mireille Cifali, op. cit., p. 107. 


\section{RÉSUMÉS}

Lors d'une enquête s'intéressant à la place de l'agressivité humaine dans les classes de l'école élémentaire, il est apparu qu'au sein des représentations de l'élève et de l'enseignant, existent des significations éclairant le passage d'une agressivité symbolisée et symbolisante, facilitant la transmission et l'appropriation du savoir, à une agressivité destructrice et déstructurante, empêchant celles-ci. À travers dessins et entretiens, sont présentées ici quelques-unes des relations existant entre les images corporelles du maitre et de l'élève, leurs représentations du fonctionnement de la classe et leurs évocations de situations, conduites ou personnalités agressives ${ }^{1}$.

Image of the body and aggressiveness in the classroom. Clinical approach of the representations of teachers and pupils in French primary schools. - During an investigation focusing on the importance of human aggressiveness in the classrooms of French primary schools, it appeared to us that, within the representation of the pupil and the teacher, there are meanings that clarify the passage from a symbolised, symbolising aggressiveness - facilitating the transmission and the appropriation of the knowledge-to a destructive, destructuring aggressiveness, preventing these processes. Through drawings and interviews we present some of the relations between corporal images of the master and the pupil, their representation of the « functioning » of the classroom and their evocations of aggressive situations, behaviours and personalities.

\section{AUTEUR}

\section{SYLVIE GÉRARD}

Sylvie GÉRARD, professeur des écoles, maître formateur, spécialisée dans l'enfance inadaptée et handicapée, occupe un poste d'ATER en Sciences de l'Éducation à l'IUFM de Paris. Membre du groupe de recherche GRAPHIES, elle termine à Paris V, sous la direction de Jean-Sébastien Morvan, une thèse intitulée La Place de l'agressivité dans le fonctionnement de la classe : approche clinique des représentations d'enseignants et d'élèves de CE1 et de CM2. De 1994 à 1996, elle a participé, comme chercheur associé, aux travaux de la DEP et de l'IHESI sur la « Violence à l'École », puis a publié en 1998 l'article « Espace-classe et agressivité à l'école élémentaire : approche clinique » dans le numéro 3 de la revue La création Sociale Société-Cultures-Imaginaires. 